\title{
Lymphatic vessels and related factors in adenoid cystic carcinoma of the salivary gland
}

\author{
Gentaro Fujita ${ }^{1,2,3}$, Sunao Sato ${ }^{1}$, Mitsunobu Kishino ${ }^{1}$, So-ichi Iwai ${ }^{2}$, Mitsuhiro Nakazawa ${ }^{2}$, \\ Satoru Toyosawa ${ }^{1}$, Yoshiaki Yura ${ }^{2}$ and Yuzo Ogawa ${ }^{1}$ \\ ${ }^{1}$ Department of Oral Pathology, Osaka University Graduate School of Dentistry, Osaka, Japan and \\ ${ }^{2}$ Department of Oral and Maxillofacial Surgery II, Osaka University Graduate School of Dentistry, \\ Osaka, Japan
}

\begin{abstract}
Adenoid cystic carcinoma of the salivary gland preferentially metastasizes to distant organs. It rarely metastasizes to lymph nodes. Recently, lymphangiogenesis has been associated with lymph node metastasis. Therefore, lymphangiogenesis in adenoid cystic carcinoma was evaluated from the number of lymphatic vessels and the expression of lymphangiogenic factors. Immunohistochemistry and molecular analysis were performed on clinical materials $(29$ cases for immunohistochemistry and 9 cases for molecular analysis). Normal submandibular gland was used as a negative control of lymphangiogenesis (10 cases for immunohistochemistry and 5 cases for molecular analysis). In adenoid cystic carcinoma, podoplanin-positive lymphatic vessels were small and often constricted, and localized to the tumor periphery. They did not have Ki67-positive endothelial cells. The lymphatic vessel density of the tumor did not exceed that of the salivary gland. By reverse transcriptase-polymerase chain reaction, adenoid cystic carcinoma and the salivary gland expressed vascular endothelial growth factor receptor-3 (VEGFR-3) similarly but VEGF-C and VEGF-D differently. Adenoid cystic carcinoma expressed VEGF-C, whereas the salivary gland expressed both VEGF$C$ and VEGF-D. VEGF-C was weak in adenoid cystic carcinoma and strong in the salivary gland. Real-time reverse transcriptase-polymerase chain reaction of VEGF-C showed that the ratio of the tumor to the salivary gland was 1 to $30(P<0.01)$. Immunohistochemistry barely detected VEGF-C in adenoid cystic carcinoma. VEGF-C was expressed faintly by the tumor cells. VEGF-C and VEGF-D were detected in the serous acinar and duct cells and in the duct contents in the salivary gland. VEGFR-3 appeared to be expressed by lymphatic vessels in both adenoid cystic carcinoma and the salivary gland. These results indicate that lymphangiogenesis does not occur in adenoid cystic carcinoma. This condition would lead to the uncommon lymphatic metastasis. Modern Pathology (2011) 24, 885-891; doi:10.1038/modpathol.2011.42; published online 18 March 2011
\end{abstract}

Keywords: adenoid cystic carcinoma; salivary gland; lymphatic vessel; podoplanin; VEGF-C; VEGF-D; VEGFR-3

It is a common clinical observation that carcinomas initially spread via the lymphatic system while sarcomas spread via the hematogenous route. Adenoid cystic carcinoma of the salivary gland accounts for about $10 \%$ of all epithelial tumors and about $23 \%$ of all carcinomas occurring in this organ., Adenoid cystic carcinoma is a highly malignant tumor and preferentially metastasizes to distant

Correspondence: Dr Y Ogawa, DDS, PhD, Department of Oral Pathology, Osaka University Graduate School of Dentistry, 1-8 Yamadaoka; Suita, Osaka 565-0871, Japan.

E-mail: ogawa@dent.osaka-u.ac.jp

${ }^{3}$ Current address: Department of Oral and Maxillofacial Surgery, Saiseikai Senri Hospital, Osaka, Japan

Received 27 September 2010; revised and accepted 8 December 2010; published online 18 March 2011 organs, especially to the lung. It rarely metastasizes to lymph nodes. ${ }^{1,2}$

Traditionally, lymphatic metastasis was thought to be a passive process by which detached tumor cells enter lymph nodes via preexisting lymphatic vessels near a primary tumor. Entry of tumor cells into lymphatic vessels would be facilitated by their thin walls and incomplete basement membrane. This view, however, has been challenged since the discovery of lymphatic markers and lymphangiogenic growth factors. Cumulative clinical and experimental evidence indicate that lymphangiogenesis, the proliferation of new lymphatic vessels from preexisting ones, is associated with lymph node metastasis. ${ }^{3}$

The immunohistochemistry of lymphatic endothelial markers such as podoplanin, lymphatic 
vessel endothelial hyaluronan receptor-1 (LYVE-1), and vascular endothelial growth factor (VEGF) receptor-3 (VEGFR-3) has demonstrated that the number of tumor-associated lymphatic vessels correlates with the presence of lymph node metastases for breast, lung, gastric, cervical, and endometrial carcinomas and head and neck squamous cell carcinoma. ${ }^{4}$ The VEGF family, which consists of VEGF-A, placental growth factor, VEGF-B, VEGF-C, and VEGF-D in mammals, has a prominent role in the formation of blood and lymphatic vessels. They bind to three tyrosine kinase receptors: VEGFR-1, VEGFR-2, and VEGFR-3. ${ }^{4,5}$ VEGF-A is a key regulator of hemangiogenesis. VEGF-A activation of VEGFR-2 on vascular endothelial cells promotes the growth of endothelial cells, prevents apoptosis of endothelial cells, and induces endothelial permeability. ${ }^{5}$ VEGF-C and VEGF-D regulate lymphangiogenesis. They bind to VEGFR-3 on lymphatic endothelial cells and stimulate the proliferation and migration of these cells. ${ }^{3,4}$ In many cancers, expression of VEGF-C or VEGF-D by tumor cells is associated with lymphatic vessel invasion and lymph node metastasis including those named above. ${ }^{3,4}$ In xenograft or transgenic mouse models of cancer, expression of VEGF-C or VEGF-D by tumor cells leads to an increase in the abundance of tumor lymphatic vessels and promotes the spread of tumor cells to lymph nodes. ${ }^{6-8}$ A soluble form of VEGFR-3 that sequesters VEGF-C and VEGF-D reduces both lymphatic vessel number and lymphatic metastasis in xenograft mouse models of cancer. ${ }^{9-11}$

The aim of this study was to clarify the reason for uncommon lymphatic metastasis of adenoid cystic carcinoma. Lymphangiogenesis in adenoid cystic carcinoma was evaluated from the number of lymphatic vessels and the expression of lymphangiogenic factors. Immunohistochemistry and molecular analysis were performed on clinical materials.

\section{Materials and methods}

\section{Tissue Samples}

Ethical approval for the use of human tissues was obtained from the Ethics Committee of Osaka University Dental Hospital and Graduate School of Dentistry. Tissue samples were obtained from 29 patients with adenoid cystic carcinoma, 13 males and 16 females, aged between 29 and 85 (median age, 58). Three patients had tumors classified as early (I and II) and 26 as late (III and IV) clinical stage. None of the patients had previously received chemotherapy or radiotherapy. Formalin-fixed, paraffin-embedded tissue blocks were made from all the samples and were used for histological analysis. In addition, unfixed frozen tissue blocks were made from eight samples and were used for molecular analysis. For negative control of lymphangiogenesis, normal salivary gland (submandibular gland) was used: 10 formalin-fixed, paraffin-embedded tissue blocks and 5 unfixed frozen tissue blocks.

\section{Immunohistochemistry}

Serial sections (5-6 $\mu \mathrm{m}$ thick) were made from paraffin-embedded tissue blocks and mounted on silane-coated glass slides (Matsunami Glass, Osaka, Japan). One section from each tissue block was stained with hematoxylin and eosin, and the others were used for immunohistochemistry. Before immunohistochemistry, antigens were unmasked by placing the sections in a $0.01-\mathrm{M}$ citrate buffer $(\mathrm{pH}$ $6.0)$ and heating them in a microwave oven $(700 \mathrm{~W}$; $5 \mathrm{~min} \times 2$ ). Immunohistochemistry was performed at room temperature unless otherwise noted. Details of the antibodies used for immunohistochemistry are listed in Table 1.

For single-labeling immunohistochemistry, the sections were treated with $0.3 \% \quad \mathrm{H}_{2} \mathrm{O}_{2}$ and then with normal goat serum (1:100; Wako, Osaka, Japan), $30 \mathrm{~min}$ each, to block endogenous peroxidase and nonspecific binding of antibodies, respectively. Thereafter, they were incubated with primary antibodies overnight at $4^{\circ} \mathrm{C}$, and then with horseradish peroxidase-conjugated polymer $\left(\right.$ EnVision $^{\mathrm{TM}}+$, Mouse/HRP or EnVision ${ }^{\mathrm{TM}}+$, Rabbit/HRP; Dako, Carpinteria, CA, USA) for $30 \mathrm{~min}$. Immunoreaction sites were visualized by treatment with $3,3^{\prime}$-diaminobenzidine tetrahydrochloride- $\mathrm{H}_{2} \mathrm{O}_{2}$ solution.

For double labeling of Ki67 and podoplanin, Ki67 immunohistochemistry was first performed as described above. Before podoplanin immunohistochemistry, the sections were microwaved as described above to block antibody cross-reactivity. ${ }^{12}$ Podoplanin immunohistochemistry was performed as above but with the use of alkaline phosphataseconjugated polymer (Histofine Simple Stain AP (MULTI); Nichirei Bioscience, Tokyo, Japan) as the second antibody and New Fuchsin Substrate System (Dako) to visualize the immunoreaction sites.

Table 1 Primary antibodies used for immunohistochemistry

\begin{tabular}{llcl}
\hline Antigen & Host animal (clone) & Dilution & Source \\
\hline Podoplanin & Mouse (D2-40) & $1: 500$ & DAKO, Carpinteria, CA, USA \\
Ki67 & Mouse (MIB1) & $1: 200$ & DAKO \\
VEGF-C & Rabbit & $1: 50$ & ABGENT, San Diego, CA, USA \\
VEGF-D & Rabbit & $1: 200$ & ABGENT \\
VEGFR-3 & Rabbit & $1: 100$ & ABGENT \\
\hline
\end{tabular}




\section{Histomorphometry}

Immunostained sections were observed with a microscope (Eclipse E600; Nikon, Tokyo, Japan) equipped with a digital camera (DXM1200F; Nikon). After scanning the section at low magnification $(\times 100)$, the area of tissue containing the greatest number of podoplanin-positive microvessels (hot spot) was selected. From the hot spot, 10 digital photographs, which were not superimposed on each other, were taken at $\times 400$ magnification (corresponding to an area of $0.062 \mathrm{~mm}^{2}$ ). The photographs were displayed on a monitor using imaging software (Photoshop; Adobe Systems, San Jose, CA, USA) and the podoplanin-positive microvessels were counted independently by two observers (SS and MK). The highest count of the 10 photographs was assigned to the lymphatic vessel density of the section (case). The mean value of the lymphatic vessel densities determined by both observers was recorded and used for further calculation.

\section{Extraction of RNA, Reverse Transcriptase-Polymerase Chain Reaction (RT-PCR), and Quantitative Real-Time RT-PCR}

Serial cryostat sections $(20 \mu \mathrm{m}$ thick) were made from frozen tissue blocks, mounted on clean glass slides (Matsunami Glass), fixed with ice-cold ethanol/acetic acid (19:1) for 3 min and washed with ice-cold diethylpyrocarbonate (DEPC; Sigma, Saint Louis, MO, USA)-treated water. The sections were stained with $0.05 \%$ toluidine blue for $10 \mathrm{~s}$, washed with ice-cold DEPC-treated water, air-dried, and used for RNA extraction.

Under a microscope (TMS; Nikon), parts of each section were cut from other parts containing unnecessary tissue such as excess connective tissue and adipose tissue with a clean scalpel and were collected in a 1.5-ml microtube. Total RNA was extracted with TRIsure (Nippon Genetics, Tokyo, Japan). First-strand cDNA was then made using a High-Capacity cDNA Reverse Transcription Kit (Applied Biosystems, Foster, CA, USA).

PCR was performed with SYBR Premix Ex Taq (Takara Bio, Tokyo, Japan). Reactions were performed using a DNA Engine PTC-200 (Bio-Rad, Tokyo, Japan) as follows: $95^{\circ} \mathrm{C}$ for $30 \mathrm{~s}$, followed by 35 cycles of $95^{\circ} \mathrm{C}$ for $5 \mathrm{~s}$ and $60^{\circ} \mathrm{C}$ for $34 \mathrm{~s}$. PCR products were separated by $1 \%$ agarose gel electrophoresis.

Real-time PCR was performed with Fast SYBR Green Master Mix Kit (Applied Biosystems). Reactions were performed using the StepOnePlus System (Applied Biosystems) as follows: $95^{\circ} \mathrm{C}$ for $20 \mathrm{~s}$, followed by 40 cycles of $95^{\circ} \mathrm{C}$ for $3 \mathrm{~s}$ and $60^{\circ} \mathrm{C}$ for $30 \mathrm{~s}$. Amplification levels were normalized to that of $\beta$-actin. Primers for PCR and real-time PCR are listed in Table 2.
Table 2 Primers used for RT-PCR and real-time RT-PCR

\begin{tabular}{|c|c|c|}
\hline Gene & Primer $\left(5^{\prime} \rightarrow 3^{\prime}\right)$ & Size, $b p$ \\
\hline VEGF-C & $\begin{array}{l}\text { F: GCCAACCTCAACTCAAGGAC } \\
\text { R: CCCACATCTGTAGACGGACA }\end{array}$ & 201 \\
\hline VEGF-D & $\begin{array}{l}\text { F: ATGGACTCTCGCTCAGCATC } \\
\text { R: ATCGGAACACGTTCACACAA }\end{array}$ & 196 \\
\hline VEGFR-3 & $\begin{array}{l}\text { F: CGTAGTCGGGGTCTTTGTAG } \\
\text { R: CTACAAAGACCCCGACTACG }\end{array}$ & 95 \\
\hline$\beta$-Actin & $\begin{array}{l}\text { F: CTCTTCCAGCCTTCCTTCCTG } \\
\text { R: CAGCACTGTGTTGGCGTACAG }\end{array}$ & 117 \\
\hline
\end{tabular}

F, forward; R, reverse.

\section{Statistical Analysis}

All data are expressed as mean \pm s.e.m. Statistical analysis was performed using Mann-Whitney's $U$-test. Differences were considered statistically significant when $P<0.05$.

\section{Results}

\section{Lymphatic Vessels}

Lymphatic vessels in cancerous and normal tissue sections were stained by monoclonal D2-40 antibody. D2-40 antibody, originally raised against M2A oncofetal antigen expressed by testicular germ cell tumors, specifically recognizes podoplanin. ${ }^{13}$ Podoplanin is expressed by lymphatic endothelial cells as well as by some nonendothelial cells including myoepithelial cells and duct cells ${ }^{13,14}$ (Figure 1a and b). As a consequence, some tumor cells were stained by the D2-40 antibody (Figure 1e). These cells, however, did not hinder the identification of lymphatic vessels.

Podoplanin-positive lymphatic vessels were localized to the extralobular connective tissue in the salivary gland (Figure 1a). In adenoid cystic carcinoma, lymphatic vessels were localized to the tumor periphery (Figure 1c). The vessels were small and often constricted so that the lumen was difficult to recognize (Figure 1d). By double-labeling immunohistochemistry, Ki67-positive endothelial cells were not observed in the lymphatic vessels in either the salivary gland or adenoid cystic carcinoma (Figure 1f). The lymphatic vessel density of adenoid cystic carcinoma did not exceed that of the salivary gland $(2.40 \pm 0.37$ vs $2.90 \pm 0.23 ; P=0.48)$. One adenoid cystic carcinoma that occurred in the minor salivary gland of the floor of the mouth had metastasized to the submandibular lymph node. The lymphatic vessel density of this carcinoma was not significantly different from that of the other carcinomas without lymph node metastasis (4.00 vs $2.34 \pm 0.38 ; P=0.36$ ).

\section{Lymphangiogenic Factors}

RNA was extracted from frozen tissue sections, and the expression of lymphangiogenic factors, VEGF-C, 

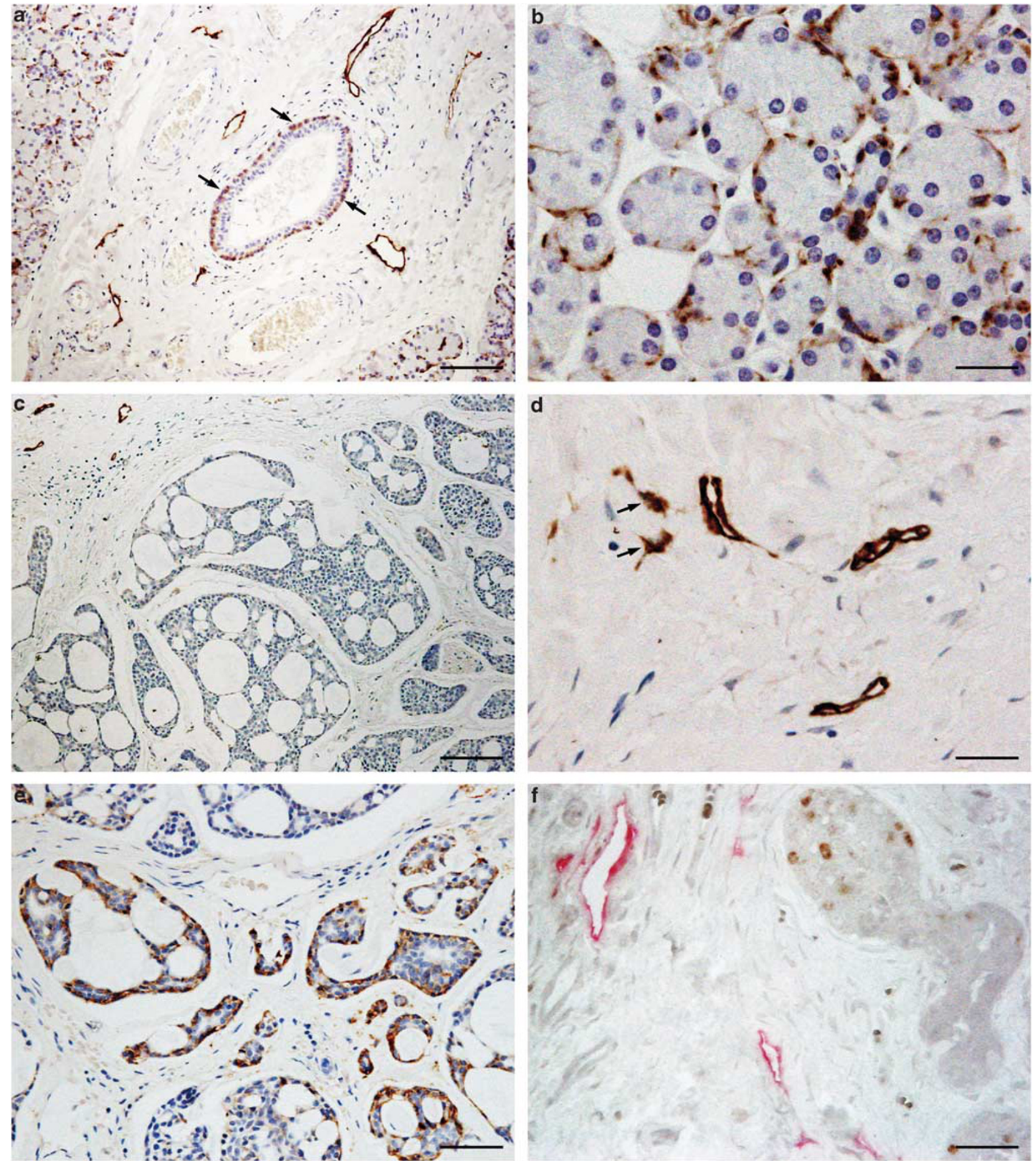

Figure 1 Immunohistochemistry for podoplanin with hematoxylin counterstaining (a-e) and for podoplanin and Ki67 without counterstaining (f). Submandibular gland $(\mathbf{a}, \mathbf{b})$ and adenoid cystic carcinoma (c-f). (a) Lymphatic vessels in the extralobular connective tissue. Note podoplanin-positive basal cells of a large duct (arrows). (b) Podoplanin-positive myoepithelial cells around acini and intercalated ducts. (c) Small lymphatic vessels are seen in the tumor periphery (upper left corner) but not in the tumor interior. (d) Higher power view of the lymphatic vessels in (c). Small lymphatic vessels are often constricted so that the lumen is difficult to recognize (arrows). (e) Some tumor cells or 'neoplastic myoepithelial cells' are podoplanin positive. (f) Ki67-positive nuclei (brown) are seen in some tumor cells but not in lymphatic vessels (red). Bars represent $100 \mu \mathrm{m}$ (a, c), $25 \mu \mathrm{m}$ (b, d), $50 \mu \mathrm{m}$ (e), and $35 \mu \mathrm{m}$ (f).

VEGF-D, and their receptor, VEGFR-3, was examined by RT-PCR. The receptor was similarly expressed by both adenoid cystic carcinoma and the salivary gland: both weakly expressed VEGFR-3 (Figure 2). The expression of the growth factors was different between adenoid cystic carcinoma and the 


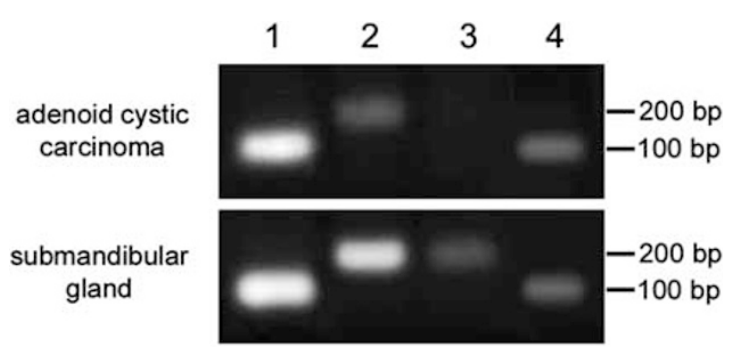

Figure 2 Representative expression pattern of mRNAs by adenoid cystic carcinoma and submandibular gland. Lane $1, \beta$-actin; lane 2, VEGF-C; lane 3, VEGF-D; lane 4, VEGFR-3.

salivary gland. Adenoid cystic carcinoma expressed only VEGF-C, whereas the salivary gland expressed both VEGF-C and VEGF-D. Expression of VEGF-C was weak in adenoid cystic carcinoma and strong in the salivary gland. When the level of VEGF-C was determined by real-time RT-PCR, the ratio of adenoid cystic carcinoma to the salivary gland was 1 to 30 ( $3.29 \pm 1.61$ vs $100.00 \pm 17.89 ; P=0.003)$.

The expression of VEGF-C, VEGF-D, and VEGFR-3 proteins was examined by immunohistochemistry. VEGF-C protein was barely detectable in adenoid cystic carcinoma. Tumor cells faintly expressed the protein (Figure 3a). In the salivary gland, VEGF-C was detected in the serous acinar cells and the duct cells (Figure 3b). In addition, VEGF-C was detected in the contents of the ducts. VEGF-D was detected in the serous acinar and duct cells in the salivary gland (Figure 3c). Sometimes, it was also detected in the duct contents (inset in Figure 3c). VEGFR-3 appeared to be expressed by the lymphatic vessels in both adenoid cystic carcinoma and the salivary gland (Figure 3d). We did not see VEGFR-3-positive blood vessels containing erythrocytes (see Discussion). We examined VEGF-C, VEGF-D, and VEGFR-3 in the tumor with lymph node metastasis by immunohistochemistry. There was no obvious difference in the expression of these proteins between this tumor and the other tumors without lymph node metastasis.

\section{Discussion}

To know the reason for the uncommon lymphatic metastasis of adenoid cystic carcinoma, lymphangiogenesis in the tumor was assessed from the number of lymphatic vessels and the expression of lymphangiogenic factors. Immunohistochemistry and molecular analysis were performed on clinical materials. We used podoplanin as the marker of lymphatic endothelial cells. Other markers such as VEGFR-3, LYVE-1, and prox-1 have been also used for immunohistochemistry of lymphatic vessels. ${ }^{15}$ VEGFR-3 and LYVE-1 are not reliable markers for discriminating between lymphatic and blood vessels. VEGFR-3 is expressed by fenestrated blood vessels in such organs as the spleen, kidney, and endocrine glands and by angiogenic blood vessels in breast cancer. ${ }^{16,17}$ LYVE-1 is expressed by sinusoidal endothelial cells in the liver. ${ }^{18}$ Thus, podoplanin and prox-1 are considered the most reliable markers; however, prox-1 is not an ideal marker for quantifying lymphatic vessels microscopically due to its nuclear localization. ${ }^{15}$

The number of podoplanin-positive lymphatic vessels represented by lymphatic vessel density was not higher in adenoid cystic carcinoma than in the salivary gland, suggesting that lymphangiogenesis was not functioning in the tumor. Indeed, endothelial cells positive for Ki67, a cell proliferation marker, were not found in the lymphatic vessels. The lymphatic vessels in adenoid cystic carcinoma were small and often constricted. There were almost no lymphatic vessels in the tumor interior. These results rather suggest the regression of lymphatic vessels.

The expression of lymphangiogenic factors and their receptor was consistent with the view that lymphangiogenesis was not functioning in adenoid cystic carcinoma. Adenoid cystic carcinoma did not express VEGF-D mRNA. It weakly expressed VEGF$\mathrm{C}$ and VEGFR-3 mRNAs. The VEGFR-3 level in adenoid cystic carcinoma was similar to that in the salivary gland, an organ with no endothelial propagation. The VEGF-C level was very low as compared with that in the salivary gland. Although the salivary gland expressed a uniquely high level of VEGF-C (see below), a low VEGF-C level in adenoid cystic carcinoma was confirmed by the fact that the growth factor was barely detectable by immunohistochemistry. VEGF-C produced in adenoid cystic carcinoma would not be enough to induce the proliferation of lymphatic endothelial cells.

Although the pathway involving VEGF-C/VEGFD/VEGFR-3 is the best validated signaling system for the establishment of lymphatic vessels, several growth factors have been implicated in lymphangiogenesis. They include VEGF-A, fibroblast growth factor (FGF)-2, platelet-derived growth factor (PDGF)-B, and angiopoietins, ${ }^{19}$ and may have an effect on the lymphatics in adenoid cystic carcinoma. VEGF-A and FGF-2 indirectly induce lymphangiogenesis by means of recruiting inflammatory cells that produce VEGF-C and VEGF-D. ${ }^{19-21}$ VEGF-A, PDGF-B, and angiopoietins are able to directly induce lymphangiogenesis via activation of the corresponding receptor (VEGFR-2, PDGFR- $\alpha$ and PDGFR- $\beta$, and Tie2, respectively) on the endothelium of lymphatic vessels. ${ }^{22-25}$ FGF-2 and PDGF-B are upregulated or overexpressed in adenoid cystic carcinoma $^{26,27}$ and thus are unlikely to have an effect on the lymphatic vessels. They would promote tumor progression by means other than by stimulation of lymphangiogenesis such as direct effects on the tumor cells and stimulation of hemangiogenesis. ${ }^{19}$ We are currently conducting a study to examine the expression of VEGF-A and angiopoietins in adenoid cystic carcinoma. 

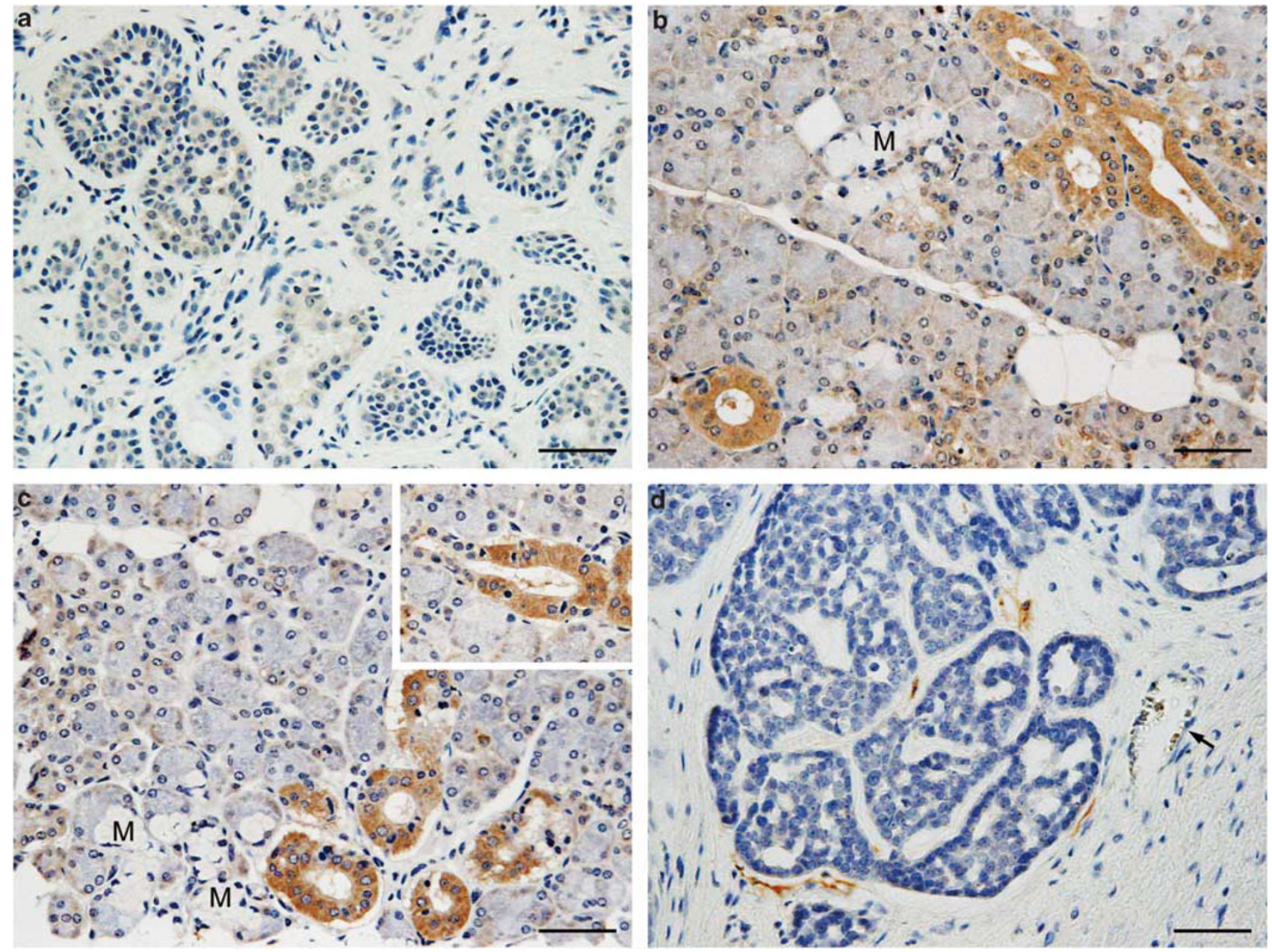

Figure 3 Immunohistochemistry for VEGF-C (a, b), VEGF-D (c), and VEGFR-3 (d) with hematoxylin counterstaining. Adenoid cystic carcinoma (a, d) and submandibular gland (b, c). (a) Faint VEGF-C staining seen in tumor cells. (b) VEGF-C seen in serous acinar and duct cells and duct contents. VEGF-C-negative mucous acinar cells (M). (c) VEGF-D seen in serous acinar cells and duct cells and sometimes in duct contents (inset). Negative mucous acinar cells (M). (d) VEGFR-3 seen in lymphatic vessels. Negative blood vessel containing erythrocytes (arrow). Bars represent $50 \mu \mathrm{m}$.

Although being dormant with respect to lymphangiogenesis, the salivary gland uniquely expressed a high level of VEGF-C mRNA. It also expressed a low level of VEGF-D mRNA. Immunohistochemistry showed that the serous acinar cells and the duct cells produced both VEGF-C and VEGF-D. The presence of VEGF-C and VEGF-D in the duct contents indicates that these molecules are secreted into saliva. The serous acinar cells and duct cells also produce VEGF-A and secrete it into saliva. ${ }^{28,29}$ Through the induction of hemangiogenesis, VEGF-A in saliva is thought to accelerate wound healing within the oral cavity. ${ }^{28,29}$ Because lymphatic vessels are indispensable for the removal of excess fluid, local debris, and inflammatory cells, wound healing would also be accelerated by VEGF-C and VEGF-D. Indeed, lymphangiogenesis by VEGF-C has accelerated wound healing in normal and diabetic animals. ${ }^{30,31}$ VEGF-C and VEGF-D undergo proteolytic processing following secretion and increase their affinity for VEGFR-3. The fully processed forms bind to VEGFR-2 as well. ${ }^{32,33}$ Thus, through activation of VEGFR-2, VEGFs may have a paracrine effect on intraglandular blood vessels. Because VEGFR-2 activation by VEGF-C induces vascular permeability, ${ }^{32}$ the molecules produced by the acinar and duct cells may permeabilize the adjoining blood capillaries, leading to the production of saliva.

In conclusion, the number of lymphatic vessels and the expression of lymphangiogenic factors indicate that lymphangiogenesis does not occur in adenoid cystic carcinoma. The absence of lymphangiogenesis would be associated with uncommon lymph node metastasis of this carcinoma. In this study, however, one carcinoma that had lymph node metastasis did not show definite lymphangiogenesis.

\section{Disclosure/conflict of interest}

The authors declare no conflict of interest. 


\section{References}

1 El-Naggar AK, Huvos AG. Adenoid cystic carcinoma. In: Barnes L, Eveson JW, Reichart P, Sidransky D (eds). WHO Classification of Tumours: Pathology and Genetics of Head and Neck Tumours. IRAC Press: Lyon, 2005, pp 221-222.

2 Regezi JA, Sciuba JJ, Jordan RCK. Oral Pathology: Clinical Pathologic Correlations, 5th edn. Saunders: St Louis, 2008, pp 207-209.

3 Stacker SA, Achen MG, Jussila L, et al. Lymphangiogenesis and cancer metastasis. Nat Rev Cancer 2002;2:573-583.

4 Pepper MS, Tille JC, Nisato R, et al. Lymphangiogenesis and tumor metastasis. Cell Tissue Res 2003;314: 167-177.

5 Ferrara N, Gerber HP, LeCouter J. The biology of VEGF and its receptors. Nat Med 2003;9:669-676.

6 Mandriota SJ, Jussila L, Jeltsch M, et al. Vascular endothelial growth factor-C-mediated lymphangiogenesis promotes tumour metastasis. EMBO J 2001;20: 672-682.

7 Stacker SA, Caesar C, Baldwin ME, et al. VEGF-D promotes the metastatic spread of tumor cells via the lymphatics. Nat Med 2001;7:186-191.

8 Skobe M, Hawighorst T, Jackson DG, et al. Induction of tumor lymphangiogenesis by VEGF-C promotes breast cancer metastasis. Nat Med 2001;7:192-198.

$9 \mathrm{He} \mathrm{Y,} \mathrm{Kozaki} \mathrm{K,} \mathrm{Karpanen} \mathrm{T,} \mathrm{et} \mathrm{al.} \mathrm{Suppression} \mathrm{of}$ tumor lymphangiogenesis and lymph node metastasis by blocking vascular endothelial growth factor receptor 3 signaling. J Natl Cancer Inst 2002;94:819-825.

10 He Y, Rajantie I, Pajusola K, et al. Vascular endothelial cell growth factor receptor 3-mediated activation of lymphatic endothelium is crucial for tumor cell entry and spread via lymphatic vessels. Cancer Res 2005;65:4739-4746.

11 Lin J, Lalani AS, Harding TC, et al. Inhibition of lymphogenous metastasis using adeno-associated virus-mediated gene transfer of a soluble VEGFR-3 decoy receptor. Cancer Res 2005;65:6901-6909.

12 Lan HY, Mu W, Nikolic-Paterson DJ, et al. A novel, simple, reliable, and sensitive method for multiple immunoenzyme staining: use of microwave oven heating to block antibody crossreactivity and retrieve antigens. J Histochem Cytochem 1995;43:97-102.

13 Schacht V, Dadras SS, Johnson LA, et al. Up-regulation of the lymphatic marker podoplanin, a mucin-type transmembrane glycoprotein, in human squamous cell carcinomas and germ cell tumors. Am J Pathol 2005;166:913-921.

14 Ordóñez NG. Podoplanin: a novel diagnostic immunohistochemical marker. Adv Anat Pathol 2006;13:83-88.

15 Van der Auwera I, Cao Y, Tille JC, et al. First international consensus on the methodology of lymphangiogenesis quantification in solid human tumours. Br J Cancer 2006;95:1611-1625.

16 Partanen TA, Arola J, Saaristo A, et al. VEGF-C and VEGF-D expression in neuroendocrine cells and their receptor, VEGFR-3, in fenestrated blood vessels in human tissues. FASEB J 2000;14:2087-2096.

17 Valtola R, Salven P, Heikkilä P, et al. VEGFR-3 and its ligand VEGF-C are associated with angiogenesis in breast cancer. Am J Pathol 1999;154:1381-1390.
18 Mouta Carreira C, Nasser SM, di Tomaso E, et al. LYVE1 is not restricted to the lymph vessels: expression in normal liver blood sinusoids and down-regulation in human liver cancer and cirrhosis. Cancer Res 2001;61:8079-8084.

19 Cao Y. Emerging mechanisms of tumour lymphangiogenesis and lymphatic metastasis. Nat Rev Cancer 2005;5:735-743.

20 Chang LK, Garcia-Cardeña G, Farnebo F, et al. Dosedependent response of FGF-2 for lymphangiogenesis. Proc Natl Acad Sci USA 2004;101:11658-11663.

21 Cursiefen C, Chen L, Borges LP, et al. VEGF-A stimulates lymphangiogenesis and hemangiogenesis in inflammatory neovascularization via macrophage recruitment. J Clin Invest 2004;113:1040-1050.

22 Gale NW, Thurston G, Hackett SF, et al. Angiopoietin-2 is required for postnatal angiogenesis and lymphatic patterning, and only the latter role is rescued by Angiopoietin-1. Dev Cell 2002;3:411-423.

23 Cao R, Björndahl MA, Religa P, et al. PDGF-BB induces intratumoral lymphangiogenesis and promotes lymphatic metastasis. Cancer Cell 2004;6: $333-345$.

24 Hong YK, Lange-Asschenfeldt B, Velasco P, et al. VEGF-A promotes tissue repair-associated lymphatic vessel formation via VEGFR-2 and the $\alpha 1 \beta 1$ and $\alpha 2 \beta 1$ integrins. FASEB J 2004;18:1111-1113.

25 Morisada T, Oike Y, Yamada Y, et al. Angiopoietin-1 promotes LYVE-1-positive lymphatic vessel formation. Blood 2005;105:4649-4656.

26 Myoken Y, Myoken Y, Okamoto T, et al. Immunohistochemical study of overexpression of fibroblast growth factor-1 (FGF-1), FGF-2, and FGF receptor-1 in human malignant salivary gland tumours. J Pathol 1996;178:429-436.

27 Vékony $\mathrm{H}$, Ylstra B, Wilting SM, et al. DNA copy number gains at loci of growth factors and their receptors in salivary gland adenoid cystic carcinoma. Clin Cancer Res 2007;13:3133-3139.

28 Pammer J, Weninger W, Mildner M, et al. Vascular endothelial growth factor is constitutively expressed in normal human salivary glands and is secreted in the saliva of healthy individuals. J Pathol 1998;186: 186-191.

29 Taichman NS, Cruchley AT, Fletcher LM, et al. Vascular endothelial growth factor in normal human salivary glands and saliva: a possible role in the maintenance of mucosal homeostasis. Lab Invest 1998;78:869-875.

30 Saaristo A, Tammela T, Timonen J, et al. Vascular endothelial growth factor-C gene therapy restores lymphatic flow across incision wounds. FASEB J 2004;18:1707-1709.

31 Saaristo A, Tammela T, circ;rkkilâ A, et al. Vascular endothelial growth factor-C accelerates diabetic wound healing. Am J Pathol 2006;169:1080-1087.

32 Joukov V, Sorsa T, Kumar V, et al. Proteolytic processing regulates receptor specificity and activity of VEGF-C. EMBO J 1997;16:3898-3911.

33 Achen MG, Jeltsch M, Kukk E, et al. Vascular endothelial growth factor D (VEGF-D) is a ligand for the tyrosine kinases VEGF receptor 2 (Flk1) and VEGF receptor 3 (Flt4). Proc Natl Acad Sci USA 1998;95: $548-553$. 